\section{Infections of scleroderma digital ulcers: A single center cohort retrospective study}

Dilia Giuggioli, ${ }^{1}$ Luca Magnani, ${ }^{2}$ Amelia Spinella, ${ }^{1}$ Gianluigi Bajocchi, ${ }^{2}$ Adalgisa Palermo, ${ }^{1}$ Federica Lumetti, ${ }^{1}$ Emanuele Cocchiara, ${ }^{2}$ Carlo Salvarani ${ }^{1,2}$

${ }^{1}$ Scleroderma Unit, University of Modena and Reggio Emilia; ${ }^{2}$ Rheumatology Unit, AUSL-IRCCS of Reggio Emilia, Italy

\begin{abstract}
Systemic sclerosis (SSc) is a complex autoimmune and up to $50 \%$ of patients develop digital ulcers. We revised fifty consecutive patients with SSc-related digital ulcers (DUs) who referred to our Scleroderma Unit. Thirty-five of them who showed clear signs of DUs infection underwent to cutaneous swab and microbiological data collection. We performed 87 cutaneous swabs overall. DUs were recurrent in $58 \%$ of the patients and multiple in $60 \%$ of patients. Fourty-four swabs (53\%) were positive for Staphylococcus aureus (13\% Methicillin-Resistant), 9 (10\%) were positive for Pseudomonas aeruginosa, and then the others less frequently isolated. Nine patients $(25 \%)$ needed hospitalization. Our data support a patient-tailored approached to DUs, particularly those infected. Selfhygiene and asepsis during dressing procedures are mandatory. Patient must be trained to avoid dangerous behaviors and reduce the risk of infection.
\end{abstract}

\section{Introduction}

Systemic sclerosis (SSc) is a complex autoimmune disease characterized by vascular damage, immune activation and fibrosis of skin and internal organs. ${ }^{1,2}$ One of the most frequent and severe SSc manifestations are digital ulcers (DUs) related to systemic vasculopathy. DUs are present in up to $50 \%$ of patients affected by SSc, ${ }^{3}$ they are often difficult to heal, recurrent and cause pain and disability. Sometimes DUs could become infected exposing patients to severe complications (osteomyelitis and gangrene) related to a worst prognosis of the disease. ${ }^{4,5}$ The management of SSc-related DUs remain challenging: they affect patient's quality of life and frequently led to work disability with a significant impact on healthcare costs. ${ }^{6}$ There is a scarce number of scientific publications on infectious complications of SSc-related DUs and any study has addressed the impact of infection on the DUs' healing process. ${ }^{6-8}$ In the present study we retrospectively revised microbiological findings from scleroderma digital ulcers and suspected bacterial infection.

\section{Materials and methods}

We revised fifty consecutive patients with SSc-related DUs who referred to our Scleroderma Unit during the last twelve months. Thirty-five of them (33 females and 2 males; mean age $65 \pm 13$ years, disease duration $14 \pm 6.7$ years), showed clear signs of infection in at least one DUs. According to the European Wound Mangement Association (EWMA) position document, ${ }^{9}$ infected DUs only were microbiologically tested by means of a cutaneous swab. We considered signs of possible infection: perilesional erythema, swelling, purulent exudates, distinctive odor and presence of pain. ${ }^{10}$ All patients fulfilled EULAR/ACR classification criteria for SSc. ${ }^{11}$ Patients were carefully evaluated based on clinical records, clinical and serological findings, DUs' characteristics and a complete wound assessment. ${ }^{3,12}$ DUs were defined as loss of both surface epithelialization and dermis; other manifestations, such as fissures or post-traumatic skin lesions, were excluded. ${ }^{3}$ We performed 87 cutaneous swabs overall at the level of the DUs' bed after removal of dried exudates, slough or dressing residue. We used sterile cotton swab, with ringer lactate-moistened tip in case of extreme wound dryness. Swab was woven side-toside and rotated at the same time, following the Levine technique. ${ }^{5,13,14} \mathrm{We}$ collected materials either from the wound bed and the wound margins. Microorganism detection was performed by means of agglutination tests with antibodies for bacterial surface specific antigens, using protein or DNA sequencing.

\section{Results}

Data of the fifty SSc patients with DUs were reported in Table 1.

Thirty-five patients had infected DUs. Most of our patients were on systemic i.v. prostanoid therapy (42 pts) alone or in combination with endothelin receptor antagonist (bosentan, 25 pts), dual endothelin receptor antagonist (macitentan, 7 pts), phosphodiesterase 5 inhibitor (sildenafil, 3 pts) and oral nifedipine (12 pts). All DUs were local-
Correspondence: Dilia Giuggioli, Rheumatology Unit Department of Internal Medicine, University of Modena and Reggio E., Policlinico di Modena Via del Pozzo, 71 41100 Modena, Italy.

Tel.: +39.059.4224053 - Fax: +39.059.4224178.

E-mail: dilia.giuggioli@unimore.it

Key words: Scleroderma, Digital Ulcers, Infections.

Contributions: DG, substantial contributions to the conception or design of the work; or the acquisition, analysis, or interpretation of data for the work; drafting the work or revising it critically for important intellectual content; LM, AS, AP, FL, EC, substantial contributions to the conception or design of the work; or the acquisition, analysis, or interpretation of data for the work; GB, drafting the work or revising it critically for important intellectual content; CS, substantial contributions to the conception or design of the work; or the acquisition, analysis, or interpretation of data for the work; Final approval of the version to be published.

Conflict of interest: The authors declare no potential conflict of interest.

Funding: None.

Ethical approval: The study obtained ethics approval.

Availability of data and material: Data and materials are available by the authors.

Please cite this article as: Giuggioli D, Magnani L, Spinella A, et al. Infections of scleroderma digital ulcers: a single center cohort retrospective study. Dermatol Rep 2021;13:9075.

Received for publication: 20 January 2021. Revision received: 26 April 2021.

Accepted for publication: 20 May 2021

This work is licensed under a Creative Commons Attribution-NonCommercial 4.0 International License (CC BY-NC 4.0).

C Copyright: the Author(s), 2021

Licensee PAGEPress, Italy

Dermatology Reports 2021; 13:9075

doi:10.4081/dr.2021.9075

ized on the acral zone of the hands (fingertips $90 \%$ of times) while only one DU was localized to toe tips. DUs were recurrent in twenty patients and in twenty-one patients we detected multiple skin lesions at a time. Regarding the thirty-five patients with infected DUs $(2 \mathrm{M} / 33 \mathrm{~F}$, mean age $65 \pm 13$ years, mean disease duration $14 \pm 11$ years), 
twenty-four of them experienced recurrent DUs and twenty-one had multiple DUs. Most of our patients had a limited SSc subtype (25pts) and anticentromere autoantibodies were the most frequently extracted (20 pts). Among the eighty-seven swabs, only four of them were negative, while eighty-three were positive for the presence of microorganisms. Forty-four (53\%) swabs were positive for Staphylococcus aureus ( $S$. aureus $) ; 9(10 \%)$ were positive for Pseudomonas aeruginosa (P. aeruginosa), 7 (8\%) for Escherichia coli (E. coli), $6(7 \%)$ for Enterococcus faecalis (E. faecalis), 5 (6\%) for Streptococcus epidermidis (S. epidermidis) and 3 (3\%) for Bacillus morganii. Interestingly, 6/44 (13.6\%) positive swabs for $S$. aureus infections showed a methicillin-resistant Staphylococcus aureus (MRSA). In addition, nine patients with infected DUs needed hospitalization. All infections responded to systemic antibiotics therapy except for MRSA which required a more aggressive and long lasting (up to fourteen consecutive days) antibiotic combination therapy in addition to surgical debridement and hygiene procedures. There is a lack of robust in vivo data for using topical antimicrobials/antiseptic for managing infected wounds. Topical antiseptic therapy could be related with toxic effects on human cells and some laboratory tests have limited their clinical use. ${ }^{15}$ Even local antimicrobial agents could induce adverse effects and they are related to allergy and bacteria resistance. ${ }^{9}$ In scleroderma patients with more severe skin involvement the use of antiseptic and antimicrobial local therapy are also related, in our experience, with excoriation in the peri wound skin, discomfort and pain.

Fecal pathogens, rapidly improved with standard therapy but were more frequently responsible for reinfections. Furthermore, our analysis showed a prevalence of $S$. aureus $(82 \%)$ or $S$. epidermidis $(8 \%)$ infection in patients with a limited SSc subtype, while MRSA infection increased significantly in patients with previous and recurring DUs infections (6/6) and/or with past history of hospitalization (6/6). Disease duration, serology, age, gender or smoking habits did not correlate with the presence of

Table 1. Clinical and serological data overview.

\begin{tabular}{|c|c|c|}
\hline Clinical and serological data & Total & Infected digital ulcers \\
\hline Systemic sclerosis patients, $\mathrm{N}$ & 50 & 35 \\
\hline Male/female & $3 / 47$ & $2 / 33$ \\
\hline Age, mean SD years & $64 \pm 13$ & $65 \pm 13$ \\
\hline Disease duration, mean SD years & $13 \pm 10$ & $14 \pm 11$ \\
\hline $\begin{array}{l}\text { Smokers/non-smokers } \\
\text { Skin subsets, N }\end{array}$ & $13 / 37$ & $10 / 25$ \\
\hline Limited cutaneous & 35 & 25 \\
\hline Diffuse cutaneous & 15 & 10 \\
\hline $\begin{array}{l}\text { Serology, } N \\
\text { Anti-Scl-70 } \\
\text { Anticentromere } \\
\text { Other antinuclear antibodies }\end{array}$ & $\begin{array}{l}12 \\
28 \\
10\end{array}$ & $\begin{array}{c}7 \\
20 \\
8\end{array}$ \\
\hline $\begin{array}{l}\text { Therapy, } \mathrm{N} \\
\text { Systemic i.v. prostanoid } \\
\text { Bosentan } \\
\text { Macicentan } \\
\text { Oral nifedipine } \\
\text { Sildenafil }\end{array}$ & $\begin{array}{c}42 \\
25 \\
7 \\
12 \\
3\end{array}$ & $\begin{array}{l}28 \\
16 \\
6 \\
6 \\
1\end{array}$ \\
\hline $\begin{array}{l}\text { Digital ulcers (\%) } \\
\text { Recurrent DUs } \\
\text { Multiple DUs } \\
\text { Fingertip ulcers } \\
\text { Hospitalization } \\
\end{array}$ & $\begin{array}{l}58 \\
60 \\
90 \\
20\end{array}$ & $\begin{array}{c}68 \\
60 \\
85.7 \\
25.7\end{array}$ \\
\hline $\begin{array}{l}\text { Swabs, N. (\%) } \\
\quad \text { Positive swabs } \\
\text { Staphylococcus aureus } \\
\text { Pseudomonas aeruginosa } \\
\text { Escherichia coli } \\
\text { Enterococcus faecalis } \\
\text { S. epidermidis } \\
\text { M. morganii } \\
\text { Others* } \\
\end{array}$ & & $\begin{array}{l}83(95) \\
44(53) \\
9(10) \\
7(8) \\
6(7) \\
5(6) \\
3(3) \\
9(10) \\
\end{array}$ \\
\hline
\end{tabular}

${ }^{*}$ C. albicans, K. pneumoniae, Citrobacter freundii, S. agalactiae, Acinetobacter ursingii, Stenotrophomonas maltophilia, Acinetobacter gyllenbergi, Serratia marcescens, Trichophyton interdigitale.

DUs infections.

Twenty-three patients got re-infected in the same DUs in an extremely variable period of time, depending on, for example: disease progression rate, immunodeficiency status, modified Rodnan skin score, disease subtype (lcSSc vs dcSSc), presence/absence of finger contraction, Raynaud phenomenon severity.

We assessed the improvement of the infection monitoring for infection clinical signs such as pain, the presence of exudate and smell. We also checking DUs dimensions such as length, width, or depth, and chromatic parameters, such as the colors of granulation or necrotic tissue in the wound bed, pallor, or erythema of surrounding skin.

\section{Discussion}

Our study reported the prevalence of DUs infections in SSc patients during a twelve-months follow-up. Among the eighty-seven swabs performed on DUs with clinical signs of infections the overall confirmation of infection was in eighty-three of them $(95.4 \%)$, with four only negative samples. Clinical assessment of DUs and particularly the evaluation of signs and symptoms of DUs infection, acquires a pivotal role highlighting that diagnosis of DUs infection is primarily a clinical skill ${ }^{3}$. Our report firstly showed pathogens' resistance in DUs infections, in particular MRSA. SSc-related DUs are per se difficult to heal and infections complications, as gangrene and osteomyelitis, are related with a worse prognosis of the disease., ${ }^{4,5,16}$ Wound infection is one of the most challenging aspects of wound management and a major contributor to healthcare costs worldwide. Nonetheless we are missing robust data about infectious complications of SSc-related DUs and about the impact of infection on DUs' healing. ${ }^{17,18}$ Exogenous microorganism could be spread by the environment, by health care personnel during dressing, by contaminated medical equipment or even by the patient him/herself. In 2012,5 we reported that fecal pathogens were found to be present in 26\% of SSc-related DUs infections but in the present paper we reported a prevalence of just the $15 \%$ ( $E$. coli + E. faecalis). Those new data are particularly intriguing because from 2012 we have been training our patients with prominent attention to hand hygiene. We strongly recommend the use of individual protection devices as disposable gloves or sterile dressing for self-medication. Those precautions have probably caused a reduction in some pathogens favoring ubiquitous ones 
like $S$. aureus. These Gram-positive bacteria is the most frequently isolated pathogen (followed by $P$. aeruginosa and $S$. epidermidis) particularly in patients on immunosuppressive therapy (mycophenolate mofetil, anti-CD 20 antibodies, azathioprine). In our database, MRSA constitute the $6 \%$ of every infection, are responsible for the $100 \%$ of our pathogen-resistant infection and are related with multiple/recurrent DUs and subsequent history of hospitalizations. Anyway, further studies are needed to better understand the role of resistant bacteria in DUs infections.

We are still lacking validated DUs' management guidelines with particular regard to those who became infected. We believe that the therapeutic approach of SSc-related DUs should be invariably patient-tailored on the basis of both general clinical conditions and careful evaluation of single skin wound. In particular, a correct therapeutical strategy should always be preceded by the assessment of each DUs, the presence of possible subclinical local complications (infections, osteomyelitis, gangrene), and/or comorbidities (district macrovascular involvement, diabetes, and other systemic disorders). ${ }^{19}$ Systemic and local treatments encompass both pathogenetic and symptomatic drugs, as well as different non-pharmacological measures. Noteworthy, chronic and procedural pain treatments using systemic and local analgesics are definitely required. ${ }^{8,20,21}$ Elevated standard of health care, self-hygiene and asepsis during procedures are mandatory as well as careful surveillance of the hospital environment. Patient must be trained to pay specific attention to avoid infections, because one of the most important reservoirs is the patients' endogenous flora (pathogens from the skin, mucous membranes and gastrointestinal tract). Our data support the need of a training of patients about the use of personal protections devices and there is clearly a need for further multicentric studies to better comprehend the role of infections in scleroderma DUs.

\section{Conclusions}

Our data support a patient-tailored approached to DUs, particularly those infected. Self-hygiene and asepsis during dressing procedures are mandatory. Patient must be trained to avoid dangerous behaviors to reduce the risk of infection due to their endogenous flora, which is, based on our data, patients' greatest reservoir.

\section{References}

1. Wigley FM. Vascular disease in scleroderma. Clin Rev Allergy Immunol 2009;36:150-75.

2. Cappelli L, Wigley FM. Management of Raynaud phenomenon and digital ulcers in scleroderma. Rheum Dis Clin North Am 2015;41:419-38.

3. Giuggioli D, Manfredi A, Lumetti F, et al. Scleroderma skin ulcers definition, classification and treatment strategies our experience and review of the literature. Autoimmun Rev 2018;17:155-64.

4. Giuggioli D, Manfredi A, Colaci M, et al. Osteomyelitis complicating scleroderma digital ulcers. Clin Rheumatol 2013;32:623-7.

5. Giuggioli D, Manfredi A, Colaci M, et al. Scleroderma digital ulcers complicated by infection with fecal pathogens. Arthritis Care Res 2012;64:295-7.

6. Hachulla E, Clerson P, Launay D, et al. Natural history of ischemic digital ulcers in systemic sclerosis: single-center retrospective longitudinal study. J Rheumatol 2007;34:2423-30.

7. Allanore Y, Denton CP, Krieg T, et al. Clinical characteristics and predictors of gangrene in patients with systemic sclerosis and digital ulcers in the Digital Ulcer Outcome Registry: A prospective, observational cohort. Ann Rheum Dis 2016;75:1736-40.

8. Giuggioli D, Manfredi A, Vacchi C, et al. Procedural pain management in the treatment of scleroderma digital ulcers. Clin Exp Rheumatol 2015;33:5-10.

9. EWMA. Hard-to-heal wounds: a holistic approach. Available from: https://ewma.org/fileadmin/user_uploa d/EWMA.org/Position documents 200 2-2008/EWMA 08 Eng final.pdf

10. Grey JE, Enoch S, Harding KG. Wound assessment. Br Med J 2006;332:285-8.

11. van den Hoogen F, Khanna D, Fransen
J, et al. Classification criteria for systemic sclerosis: an American College of Rheumatology/European League against Rheumatism collaborative initiative. Arthritis Rheum 2013;65:273747.

12. Abraham S, Steen V. Optimal management of digital ulcers in systemic sclerosis. Ther Clin Risk Manag 2015;11: 939-47.

13. Sibbald RG, Schultz G, Keast DH. Preparing the wound BED 2003: Focus on infection and inflammation. Available from: www.nhscrd.york. ac.uk. (2003).

14. Leaper DJ, Schultz G, Carville K, et al. Extending the TIME concept: what have we learned in the past 10 years? Int Wound J 2012;9:1-19.

15. Leaper DJ. Eusol. Br Med J 1992;304: 930-1.

16. Mihai C, Landewé R, Van Der Heijde $\mathrm{D}$, et al. Digital ulcers predict a worse disease course in patients with systemic sclerosis. Ann Rheum Dis 2016;75:6816.

17. Morrisroe K, Stevens W, Sahhar J, et al. Digital ulcers in systemic sclerosis: Their epidemiology, clinical characteristics, and associated clinical and economic burden. Arthritis Res Ther 2019;21:299.

18. Matucci-Cerinic M, Krieg T, Guillevin $\mathrm{L}$, et al. Elucidating the burden of recurrent and chronic digital ulcers in systemic sclerosis: long-term results from the DUO Registry. Ann Rheum Dis 2016;75:1770-6.

19. Hughes M, Herrick AL. Digital ulcers in systemic sclerosis. Rheumatology 2017;56:14-25.

20. Giuggioli D, Manfredi A, Colaci M, et al. Oxycodone in the Long-Term Treatment of Chronic Pain Related to Scleroderma Skin Ulcers. Available from: https://academic.oup.com/painmedicine/article-abstract/11/10/1500/ 1866040

21. Ughi N, Crotti C, Ingegnoli F. Effectiveness and safety of oxycodone/naloxone in the management of chronic pain in patients with systemic sclerosis with recurrent digital ulcers: Two case reports. Clin Interv Aging 2016;11:307-11. 